\title{
SEARCH FOR NONTHERMAL X-RAYS FROM SUPERNOVA REMNANT SHELLS
}

\author{
R. PETRE, J. KEOHANE, U. HWANG, G. ALLEN, E. GOTTHELF \\ $N A S A / G S F C$ \\ Greenbelt, MD 20771 USA
}

\section{Introduction}

The suggestion that the shocks of supernova remnants (SNR's) are cosmic ray acceleration sites dates back more than 40 years. While observations of nonthermal radio emission from SNR shells indicate the ubiquity of $\mathrm{GeV}$ cosmic ray production, there is still theoretical debate about whether SNR shocks accelerate particles up to the well-known "knee" in the primary cosmic ray spectrum at $\sim 3,000 \mathrm{TeV}$. Recent X-ray observations of SN1006 and other SNR's may have provided the missing observational link between SNR shocks and high energy cosmic ray acceleration. We discuss these observations and their interpretation, and summarize our ongoing efforts to find evidence from X-ray observations of cosmic ray acceleration in the shells of other SNR's.

\section{ASCA and the Mystery of SN1006}

The X-ray spectrum of SN1006 was for a long time a puzzle. Contrary to the prevailing wisdom that the X-ray emission in shell like SNR's is produced by the shock heating of ISM material and metal-rich ejecta (and thus should be dominated by lines), the integrated spectrum of SN1006 above 1 $\mathrm{keV}$ is featureless (Becker et al. 1980), and best fit by a power law. While a model was put forward suggesting the $\mathrm{X}$-rays arise as synchrotron radiation from electrons accelerated to high energy in the shock (Reynolds \& Chevalier 1981), the generally accepted explanation was that they arise from a thermal plasma in an extreme state of ionization nonequilibrium (Hamilton et al. 1986). The ASCA observations solved this mystery, and in so doing produced the first strong evidence for the production of high energy cosmic rays in SNR shocks. Spatially resolved ASCA spectra showed that 
throughout most of the remnant, the spectrum is dominated by emission lines, with inferred metal abundances characteristic of Type Ia SN ejecta. In contrast, the spectra of the bright $\mathrm{NE}$ and $\mathrm{SW}$ rims are dominated by a power law component with $\alpha \sim 2$. Thus we can understand SN1006 as being an ordinary thermal remnant, whose spectrum above $1 \mathrm{keV}$ is dominated by a second, nonthermal component that is restricted to the bright limbs. The most reasonable model for this is synchrotron emission from electrons accelerated within the shock. At these highly relativistic energies, shock acceleration processes make no distinction between positively and negatively charged particles; thus we can infer the presence of high energy protons and nucleons as well, and conclude that cosmic ray acceleration occurs in the shell of SN1006. Reynolds (1997) has provided a shock acceleration model that reproduces both the broad band (radio through X-ray) spectrum of SN1006 and the X-ray morphology.

\section{Nonthermal X-rays from the Shells of Other SNR}

At least three other SNR's are now known to have nonthermal emission associated with their shock fronts: Cas A, G347.5-0.5, and IC 443. The existence of nonthermal tails in this many remnants (coupled with the discovery of $\mathrm{TeV} \gamma$-rays from SN1006 - Tanimori et al. 1997), firmly establishes SNR's as a source of high energy cosmic rays.

Cas A: The striking morphological similarity between the ASCA 4-8 $\mathrm{keV}$ continuum map of Cas A and the radio image, and its contrast with the maps in all other X-ray bands, suggested that the hard continuum might arise from nonthermal processes (Holt et al. 1995). Recent RXTE and SAX spectra show a nonthermal spectrum that extends beyond $100 \mathrm{keV}$ (Allen et al. 1997; Favata et al. 1997). This is almost certainly synchrotron emission from shock accelerated electrons.

G347.5-0.5: Koyama et al. (1997) found that the shell-like supernova remnant G347.5-0.5 (= RX J1713.7-3946), discovered in the ROSAT AllSky Survey, has a featureless power law spectrum with $\alpha \sim 2.5$. Its similarity to SN1006 led Koyama et al. to propose that G347.5-0.5 is the second example of a shell-like SNR dominated by synchtron emission from shock acclerated electrons.

IC 443: ASCA imaging reveals that the hard component is localized to the region where the shock is interacting most strongly with a foreground molecular cloud (Keohane et al. 1997). The shock velocity in IC 443 is too low to support the production of $\mathrm{TeV}$ particles in the way they are produced in SN1006. Keohane et al. propose instead that the synchrotron electrons are produced by enhanced shock acceleration arising directly from the cloud/shock collision (Jones \& Kang 1993). 


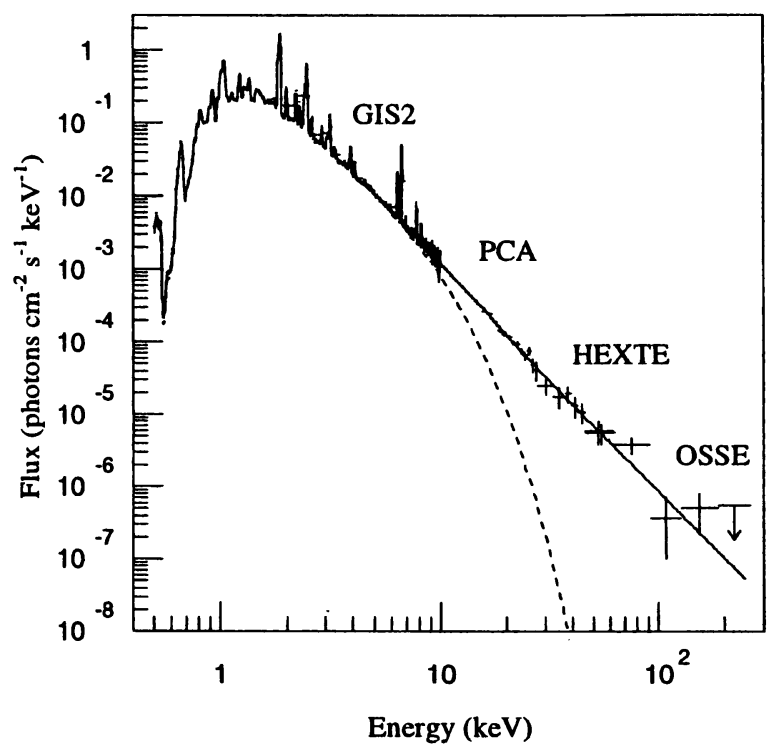

Figure 1. Broad band unfolded X-ray spectrum of Cas A, showing hard power law tail beyond $100 \mathrm{keV}$ (from Allen et al. 1997)

We have embarked on a systematic search through the ASCA data for nonthermal tails in young Galactic SNR's. Our preliminary results for some remnants (W49B, 3C 397, Kepler, Tycho) have been compiled in Table 1 , along with the remnants for which results have been published. While one might expect some correlation between the existence of a nonthermal shell component and age (shock velocity), radio brightness (magnetic field strength, electron density), or a X-ray/radio morphology match, no clear pattern has yet emerged.

\section{The LMC Remnants}

The young, X-ray luminous remnants in the Large Magellanic Cloud allow for the study of a full population of SNR's without the biases introduced within the Galaxy by distance and column density. We have systematically searched the spectra of the luminous LMC remnants observed by ASCA for evidence of hard tails. In general, remnants with small diameters have hard components and those with large diameters (e.g., N49B) do not. This suggests that the mechanism producing the tails is restricted to the younger remnants. While the presence of an Fe $\mathrm{K}$ line at $6.7 \mathrm{keV}$ demonstrates that the hard X-ray emission in some remnants (N103B, N132D, N63A) is at least partly thermal, in other remnants ()no Fe $\mathrm{K}$ line is present, suggesting that the tail could be nonthermal. The spectral index of these tails is consistent with that found in SN1006 and Cas A. If the hard X-ray 
emission in the LMC remnants arises from the same mechanism operating in the young Galactic remnants, then it provides the first evidence of high energy cosmic ray production in SNR's outside the Milky Way, and allows an opportunity to track the evolution of the cosmic ray production process.

Table 1. Preliminary results for Galactic SNR.

\begin{tabular}{|c|c|c|c|c|c|c|}
\hline Object & $\begin{array}{l}\text { Age } \\
(\mathbf{k y})\end{array}$ & $\begin{array}{l}\text { Hard } \\
\text { Tail? }\end{array}$ & $\begin{array}{l}\text { Radio/X-ray } \\
\text { Match? }\end{array}$ & Type & $\begin{array}{l}\text { High } \\
\mathrm{L}_{R} ?\end{array}$ & Result \\
\hline Cas A & 0.3 & $\mathbf{Y}$ & $\mathbf{Y}$ & II & $\mathbf{Y}$ & $\begin{array}{l}\text { Hard } X \text { cont. has } \alpha \sim 2 \text { to } 100 \mathrm{keV} \\
\text { Hard continuum morphology matches radio }\end{array}$ \\
\hline Kepler & 0.4 & - & $\mathbf{Y}$ & II? & - & $\begin{array}{l}\text { NT component improves fit } \\
\text { No strong constraint on } \alpha \\
\text { or nonthermal flux fraction from ASCA }\end{array}$ \\
\hline Tycho & 0.4 & $\mathbf{Y}$ & - & Ia & $\mathbf{Y}$ & nonthermal $\mathrm{L}_{x}<0.3 \mathrm{~L}_{1006}$ from ASCA \\
\hline SN1006 & 1.0 & $\mathbf{Y}$ & $\mathbf{Y}$ & Ia & $\mathrm{N}$ & Obvious nonthermal shell; $\alpha \sim 2$ to $\sim 20 \mathrm{keV}$ \\
\hline G347.5 & $\sim 1.0$ & $\mathbf{Y}$ & $?$ & II? & $\mathrm{N}$ & Nonthermal - "SN1006 twin" $\alpha \sim \alpha_{1006}$ \\
\hline $3 C 397$ & $\sim 1.0$ & $\mathbf{Y}$ & $\mathrm{Y}$ & II? & Y & Hard X-ray morphology matches radio \\
\hline W49B & $1-3$ & $\mathbf{Y}$ & $Y$ & $?$ & $Y$ & $\begin{array}{l}\text { Hard X-ray has } \alpha \sim \alpha_{1006} \\
\text { Hard X-ray morphology matches radio }\end{array}$ \\
\hline IC 443 & $1-4$ & $\mathbf{Y}$ & - & II & $Y$ & $\begin{array}{l}\text { Hard } X \text { isolated; } \alpha \sim \alpha_{1006} \\
\text { near } M C \text { interaction site }\end{array}$ \\
\hline
\end{tabular}

\section{Summary of Current Results}

The search for nonthermal components and hence cosmic ray acceleration in the shells of SNR has barely begun. Nevertheless, we can already formulate some conclusions. (1) The hard X-ray flux in at least four SNR's is dominated by synchrotron emission from their shells. (2) Several other SNR's have properties that suggest the presence of nonthermal shell emission. Among these are Tycho, Kepler, W49B and 3C 397. (3) A systematic search through the archival ASCA spectra of LMC remnants produces suggests that the strength of the hard component, whether thermal or nonthermal, diminishes with SNR age.

\section{References}

Allen, G.E., et al. 1997 Ap. J. Lett. 487, L97.

Becker, R.H., et al., 1980 Ap. J. Lett. 235 , L5.

Hamilton, A.J.S., Sarazin, C.L., \& Szymkowiak, A.E., 1986, Ap. J. 300, 698.

Holt, S.S., et al., 1994, Publ. Astron. Soc. Japan 46, L151.

Jones, T.W., \& Kang, H., 1993, Ap. J. 402, 560.

Keohane, J.W., et al., 1997, Ap. J. 484, 350.

Koyama, K., Petre, R., Gotthelf, E.V., Hwang, U., Matsuura, M., Ozaki, M., \& Holt, S.S., 1995, Nature 378, 255.

Koyama, K., et al., 1997, Publ. Astron. Soc. Japan 49, L7.

Reynolds, S.P., 1996, Ap. J. Lett. 459, L13.

Reynolds, S.P., \& Chevalier, R.A., 1981, Ap. J. 245, 912.

Tanimori, T., et al., 1997, IAU Circular 6706. 